\title{
Symmetric Random Walks on Regular Tetrahedra, Octahedra and Hexahedra
}

\author{
Jyotirmoy Sarkar ${ }^{1}$ and Saran Ishika Maiti ${ }^{2}$ \\ ${ }^{1}$ Indiana University-Purdue University Indianapolis, USA \\ ${ }^{2}$ Visva-Bharati, Santiniketan, India
}

Third Revision: 2017 January 08

\begin{abstract}
We study a symmetric random walk on the vertices of three regular polyhedra. Starting from the origin, at each step the random walk moves, independently of all previous moves, to one of the vertices adjacent to the current vertex with equal probability. We find the distributions, or at least the means and the standard deviations, of the number of steps needed (1) to return to origin, (2) to visit all vertices, and (3) to return to origin after visiting all vertices. We also find the distributions of (i) the number of vertices visited before return to origin, (ii) the last vertex visited, and (iii) the number of vertices visited during return to origin after visiting all vertices.
\end{abstract}

Key Words and Phrases: Absorbing state, Cover time, Dual graph, Eventual transition, One-step transition, Platonic solids, Recursive relation, Return time, Symmetry

2010 Mathematics Subject Classifications: primary 60G50; secondary 60G40

\section{Introduction}

For over two millennia, Platonic solids (also called regular polyhedra) have been regarded as fascinating mathematical objects. A Platonic solid is a three dimensional convex space bounded by congruent regular polygonal faces; and at each of its vertices an equal number of faces meet. Depicted in Fig. 1, are the five Platonic solids - tetrahedron, hexahedron (cube), octahedron, dodecahedron and icosahedron. See Euclid [3], for example, for an elementary explanation as to why there are exactly five Platonic solids. Also see Cromwell [2] for an exposition of their mathematical beauty and symmetry. The Greek philosopher Plato (about 428-347 BCE) - after whom these solids are named - theorized that these five solids correspond to the five classical elements - fire, earth, air, ether and water.

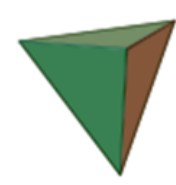

Tetrahedron

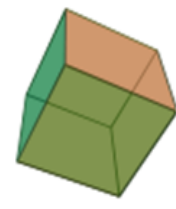

Cube

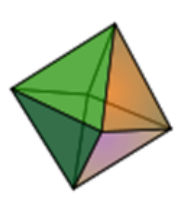

Octahedron

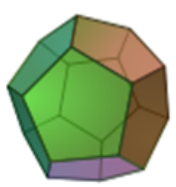

Dodecahedron

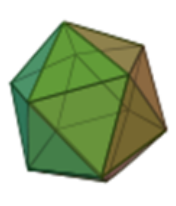

Icosahedron

Figure 1: The five Platonic solids

In this paper we study symmetric random walks (SRW) on the first three Platonic solids - tetrahedron, octahedron and hexahedron; and we leave the remaining two Platonic solids for later studies. At the outset, we must mention that we are considering regular polyhedra only for aesthetic reasons. However, regularity as such has no bearing on

\footnotetext{
${ }^{1}$ Corresponding author email: jsarkar@iupui.edu (J. Sarkar)
} 
the results of this paper, because they remain valid even for non-regular solids that are topologically equivalent.

We label the vertices of a regular polyhedra according to the following convention: The vertices of a regular tetrahedron are denoted by $\{0,1,2,3\}$. It does not matter which vertex gets what label since all pairs of vertices are connected by edges. The vertices of a regular octahedron are denoted by $\{0,1,2,3,4,5\}$ in such a way that three pairs of opposite vertices $(0,5),(1,4),(2,3)$ not directly connected by edges. The vertices of a regular hexahedron are denoted by $\{0,1,2,3,4,5,6,7\}$ such that Vertices $1,2,3$ are adjacent to Vertex 0, Vertices 4,5,6 are adjacent to Vertex 7, and four pairs of opposite vertices $(0,7),(1,6),(2,5),(3,4)$ are not directly connected by edges. See Fig. 2.
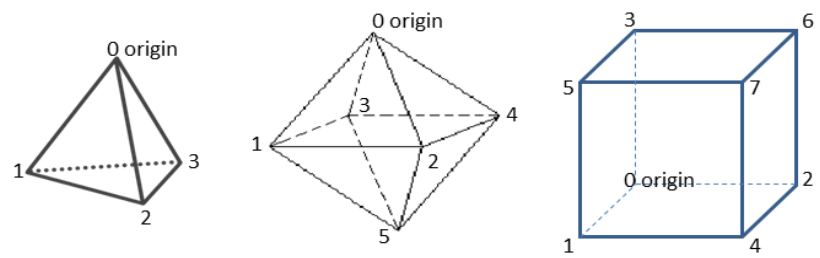

Figure 2. Numbering the vertices of a tetrahedron, an octahedron and a hexahedron

Let $t=0,1,2, \ldots$ denote the time epochs when the random walk $(\mathrm{RW})$ moves from one vertex to an adjacent vertex. Let $X(t)$ denote the location of the RW at time $t$. Without loss of generality, the RW starts at Vertex 0 ; that is, $X(0)=0$.Vertex 0 is called the origin. Thereafter, at each successive epoch, from the current vertex the RW moves to one of the adjacent vertices with equal probability. This is why the RW is called symmetric. Also, as the RW continues on and on, the successive moves are independent. Consequently, $\{X(t): t \geq 0\}$ is a discrete-time Markov chain on a discrete state space consisting of all vertices. Because the states are connected and finitely-many, the chain is irreducible.

We study the probability distributions of the times until some predefined events happen. Taking a cue from Sarkar (2006), we identified three such events: (1) Return to the origin, (2) Visiting all vertices at least once, and (3) Return to the origin after visiting all vertices. The epochs when these events occur are respectively called the return time $T_{R}$, the cover time $\bar{T}$, and the additional time to return after visiting all vertices ${ }_{L} T_{R}$, where $L=X(\bar{T}) \neq 0$. Since $X\left(T_{R}\right)=0$ and $X\left({ }_{L} T_{R}\right)=0$, it follows that $T_{R} \neq \bar{T}$.

Also, we study the probability distributions of $N_{R}, L$, and ${ }_{L} N_{R}$ defined as follows: (i) $N_{R}$ is the number of non-origin vertices visited until the RW returns to origin; that is, $N_{R}$ is the number of distinct elements of $\left\{X(1), X(2), \ldots, X\left(T_{R}-1\right)\right\}$; (ii) $L=X(\bar{T})$ is the last vertex visited; that is, the location of the RW when all vertices have been visited at least once; and (iii) ${ }_{L} N_{R}$ is the number of vertices visited while the RW returns to origin after visiting all vertices; that is, ${ }_{L} N_{R}$ is the number of distinct elements of $\left\{X(\bar{T}), X(\bar{T}+1), \ldots, X\left({ }_{L} T_{R}-1\right)\right\}$.

The study of RWs on the vertices of graphs has a rich literature. Gödel and Jagers [4] studied a RW on a connected graph; and found expressions for the expected recurrence time, first passage time and symmetrized first passage time (also known as commuting time). Letac and Takács [6] studied the limiting probability that an SRW visits a particular vertex of a dodecahedron; and then they related the result to the spectrum of a certain finite-dimensional Banach algebra. In [9] van Slijpe established that, for distance-regular graphs (which include Platonic solids), the mean passage times from one vertex to another and vice versa are equal; and in [10] they extended the result to vertex-transitive graphs. 
Wildberger [11] described the hypergroup structures (or the character table) associated with any distance transitive graph (which include Platonic solids); and using commutative harmonic analysis, he found the probability of the RW returning to the origin after $n$ steps. Sarkar [8] studied all six questions mentioned above in the context of an asymmetric random walk on a polygon. These same questions are answered by Maiti and Sarkar [7] for the SRW on a finite linear path or on a finite cyclic network.

Our objective in this paper is two fold: First, to all six questions stated above, we present elementary solutions. Second, we wish to inspire young mathematicians to fillin some details of derivations and extend this work to study SRW on other interesting graphs. Other researchers might also find our results useful.

Let us introduce some terminologies and notation: The distance between two vertices $i$ and $j$ is the number of edges on the shortest path connecting $i$ and $j$. Let ${ }_{i} T_{j}$ denote the number of steps the RW, starting from Vertex $i$, takes to reach Vertex $j$ for the first time. That is,

$$
\left.{ }_{i} T_{j}=\min \{t>0: X(s+t)=j), X(s)=i \text { for some } s>0\right\}
$$

with the usual convention of taking $\infty$ as the minimum of the empty set. In fact, the irreducibility of the chain on a finite number of states ensures that ${ }_{i} T_{j}$ so defined is actually finite with probability one. So are all the time variables considered in this paper. For all regular polyhedra, by renumbering the vertices, if necessary, we have $T_{R}={ }_{0} T_{0}=1+{ }_{1} T_{0}$, and ${ }_{L} T_{R}={ }_{L} T_{0}$. Let us denote the mean, the mean square and the variance of ${ }_{i} T_{j}$, respectively by ${ }_{i} e_{j}=E\left[{ }_{i} T_{j}\right],{ }_{i} s_{j}=E\left[{ }_{i} T_{j}^{2}\right]$, and ${ }_{i} v_{j}=V\left({ }_{i} T_{j}\right)={ }_{i} s_{j}-{ }_{i} e_{j}{ }^{2}$; where $E$ denotes expectation and $V$ denotes variance.

Here are the steps involved in answering each of the six questions about the RW on Platonic solids: (1) We draw an appropriate transition diagram. In this diagram, the nodes represent a triplet consisting of the origin, the vertex where the RW currently is, and the set of vertices the RW has visited already. (2) If the RW can go from one node to another (or itself) in one step, then we draw an arc connecting the nodes; and we specify the transition probability on the arc. (3) Whenever entry into a node signifies that the mission has been accomplished (for whichever purpose the diagram is drawn), we call that node an absorbing state. (4) We eliminate the loops (arcs going from a node to itself) and exchanges (arcs going directly to and fro between two nodes), and simultaneously we revise the conditional transition probabilities of reaching a node that involves one more visited vertex. (5) For the random variable (RV) necessary to answer the question, we obtain the entire probability distribution when possible; otherwise, we report only the mean and the standard deviation (SD).

Henceforth, in the context of a particular transition diagram, we reserve the word 'vertex' to mean a point where at least three of the edges of a Platonic solid meet, and the word 'node' to refer to a triplet of the origin, the current vertex and the set of vertices already visited.

Whereas the answers to the six questions for a tetrahedron are rather straight-forward, we still document the detailed solutions in order to develop the notation and to illustrate the techniques. In Sections 2-4, we study the SRW on a tetrahedron, an octahedron and a hexahedron respectively. In all sections, we answer the Questions in the order 1, (i), (ii), 2, 3 and (iii). In Section 5, we summarize the paper; and we suggest several directions of future research. 


\section{SRW on a Tetrahedron}

The graph of a tetrahedron shown in Fig. 2 is the complete graph $K_{4}$ on four vertices. This completeness property makes the answers to our six questions relatively simple.

\subsection{Return to Origin on a Tetrahedron}

To study $T_{R}$ on a tetrahedron, we depict in Fig. 3 a simple transition diagram involving only two nodes, which are defined by the distance of the current vertex from the origin. The set of already visited vertices plays no role here.
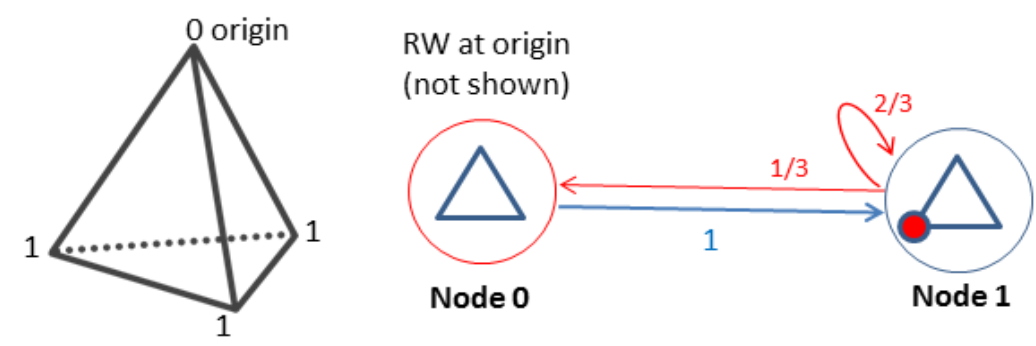

Figure 3. Distance from the origin defines these nodes. One-step transition probabilities between nodes help study $T_{R}$ for a tetrahedron

Starting from Vertex 0, the RW surely goes to a non-origin vertex, which we can renumber as Vertex 1. If the RW moves from Vertex 1 to Vertex 0, which happens with probability $1 / 3$, then ${ }_{1} T_{0}=1$. But if the RW moves from Vertex 1 to either Vertex 2 or Vertex 3, then ${ }_{1} T_{0}=1+{ }_{1} T_{0}{ }^{\prime}$, where ${ }_{1} T_{0}^{\prime}$ is a RV independent and identically distributed (IID) as ${ }_{1} T_{0}$. This is because we can renumber the current vertex as Vertex 1 , and the remaining two non-origin vertices as Vertex 2 and Vertex 3 (in either of two possible ways). In other words, from Node 1 the RW moves to Node 0 with probability $1 / 3$, and it remains at Node 1 with probability $2 / 3$. Hence, ${ }_{1} T_{0}$ is distributed as a geometric $(1 / 3)$ RV, which has mean 3 and variance 6 . Thus we have proved the following result.

Proposition 2.1.1 For a tetrahedron, the time $T_{R}$ to return to origin is one more than a geometric $(1 / 3) R V$. Hence, $T_{R}$ has mean 4, variance 6 and $S D 2.4495$.

To study $N_{R}$, we need a different transition diagram in which the nodes keep track of the entire triplet consisting of the origin, the current vertex and the set of vertices already visited. Fig. 4(a) shows the one-step transitions until return to origin (which is the mission here). The conditional probability on each arrow is $1 / 3$. In this diagram, and in all following diagrams, we use the following convention: (i) the origin is always hidden from view; (ii) an empty circle denotes a vertex already visited; (iii) a filled circle denotes the current location of the RW; and (iv) a vertex with no circle at all indicates that the vertex has not been visited yet. Then in Fig. 4(b), we draw the eventual transitions (after eliminating the loops, which are arrows going from a node to itself), together with conditional probabilities of eventual transitions written on each arrow.

Let us explain how to interpret the nodes and transitions in Fig. 4(a). We leave it to the reader to interpret similarly all other figures. The RW surely leaves the origin to go to Vertex 1 (renamed, if necessary). Thus, Node 0 surely leads to Node 1 . Then the RW goes back to the origin in one step without visiting any other vertex with probability $1 / 3$ (giving rise to Node $1 \mathrm{R}$ ), or it visits a new vertex with probability $2 / 3$. This new 


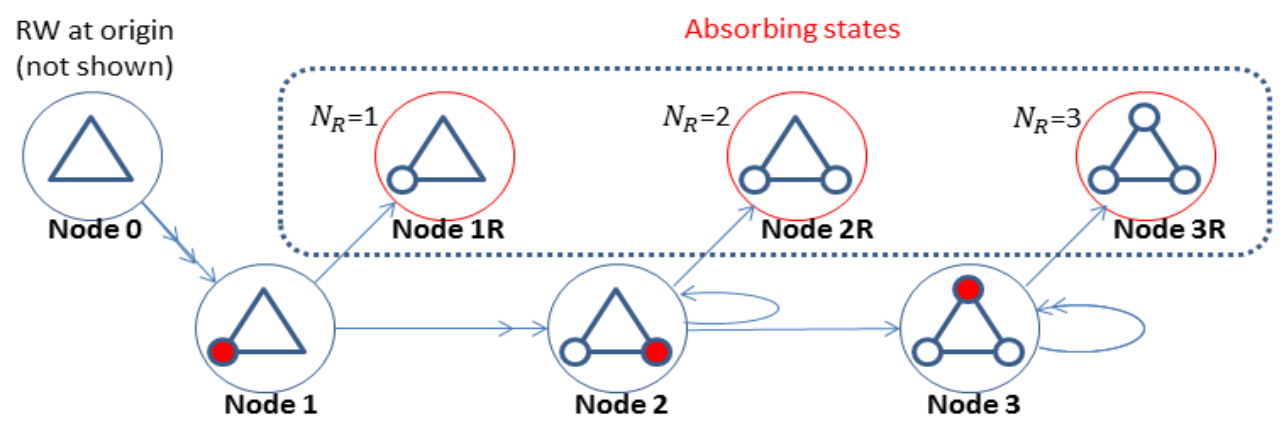

Figure 4(a): One-step transitions until return to origin, help study $N_{R}$ for a tetrahedron (conditional probability is $1 / 3$ on each arrow)

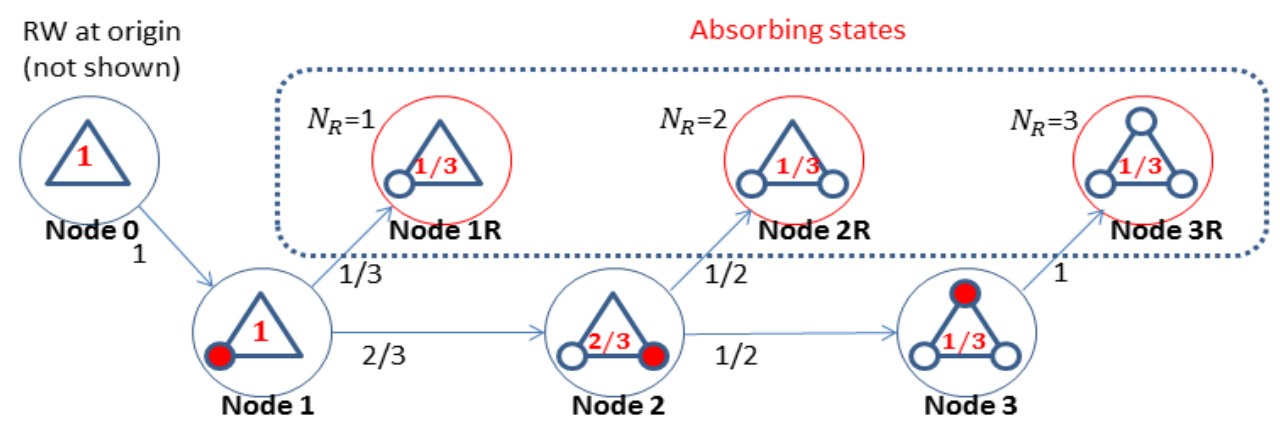

Figure 4(b): Eventual transitions until return to origin, help study $N_{R}$ for a tetrahedron (conditional probability on each arrow, ultimate probability inside each node)

vertex we renumber as Vertex 2, if necessary, and the remaining vertex we renumber as Vertex 3, giving rise to Node 2. From Vertex 2, in one step the RW goes back to either the origin, or Vertex 1, or Vertex 3 with probability $1 / 3$ each. Should it go to Vertex 1 , we interchange the numbering of Vertices 1 and 2, and we declare that after one step the RW is still at the same Node 2. Thus, eventually the RW goes either to the origin (giving rise to Node $2 \mathrm{R}$ ), or to Vertex 3 (giving rise to Node 3 ) with conditional probability $1 / 2$ each. From Vertex 3, in one step the RW goes either to the origin with probability $1 / 3$, or to Vertex 1 or 2 with probability $2 / 3$. In this final case, we renumber the current vertex as Vertex 3 (and the two non-origin vertices as Vertex 1 and Vertex 2); and we declare that after one step the RW remains in the same Node 3 (with probability 2/3). Thus, eventually (after a geometric(1/3) amount of time) the RW surely leaves Vertex 3 to go to the origin (giving rise to Node $3 \mathrm{R}$ ).

In Fig. 4(b), we show the eventual transitions, together with the conditional probability on each arrow. We obtain the probability of reaching any particular node, by multiplying the conditional probabilities along the directed pathway from Node 0 to that desired node. Specifically, the absorbing nodes $1 \mathrm{R}, 2 \mathrm{R}$ and $3 \mathrm{R}$ (where $N_{R}$ equals $1,2,3$ respectively) are reached by the RW with probability $1 / 3$ each. Thus, we have proved the following result.

Proposition 2.1.2 For a tetrahedron, $N_{R}$ is uniformly distributed over $\{1,2,3\}$. Hence, $N_{R}$ has mean 2 , variance $2 / 3$ and $S D 0.8165$.

Remark 1. Even before we study the distribution of the cover time $\bar{T}$, we can determine which event happens first - returning to origin or visiting all vertices. From Section 1, recall that $T_{R} \neq \bar{T}$. Then note that the event $\left\{T_{R}>\bar{T}\right\}$ means that the RW visits all vertices before returning to origin; that is, $\left\{N_{R}=3\right\}$. So, $P\left\{T_{R}>\bar{T}\right\}=P\left\{N_{R}=3\right\}=$ $1 / 3$. Hence, $P\left\{T_{R}<\bar{T}\right\}=P\left\{N_{R}<3\right\}=2 / 3$. 


\subsection{Visiting All Vertices on a Tetrahedron}

We next study the cover time $\bar{T}$ of a tetrahedron, and the last vertex to be visited $L=X(\bar{T})$. It might help the reader to imagine that there is a cookie at each vertex. A cookie monster starts at Vertex 0 , and eats the cookie there. Then in the next step it goes to an adjacent vertex with probability $1 / 3$, and eats the cookie there. Then in the next step it goes to the next neighboring vertex with probability $1 / 3$, and eats the cookie there, if there is a cookie there; or it stays hungry. It continues in this manner until all four cookies are eaten. Then $\bar{T}$ denotes the number of steps needed until all four cookies are eaten; and $L=X(\bar{T})$ denotes which cookie is eaten the last. Invoking symmetry, we arrive at the following result.

Proposition 2.2.1 For a tetrahedron, starting from Vertex 0 (origin), the Vertex L, which is visited the last, is uniformly distributed over $\{1,2,3\}$.

To study $\bar{T}$ we only need to keep track of the pattern of visited vertices and the current vertex; and we can safely forget the original labeling of the vertices (including the origin). In fact, it suffices to keep track of the number of (non-origin) vertices already visited. In Fig. 5, we draw the one-step transitions until all vertices are visited at least once. Each arrow represents a conditional probability of $1 / 3$. Again, the origin is not shown; etc.

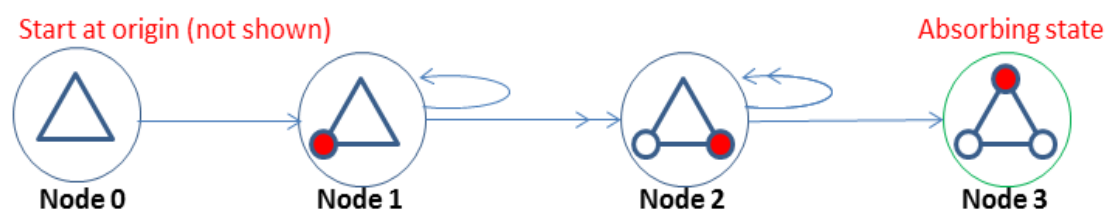

Figure 5: One-step transitions until visit all vertices, help study $\bar{T}$ for a tetrahedron (probability is $1 / 3$ on each arrow). Eventually reaches absorbing state

The nodes in this transition diagram are labeled as $0,1,2,3$; among which Node 3 is the only absorbing state, because it indicates that all vertices have been visited at least once. The interpretation of nodes and transitions in Fig. 5, and henceforth in all figures, is similar to the interpretation given for Fig. 4(a). We leave it to the reader to provide the details.

For $i=0,1,2$, let ${ }_{i} \bar{T}$ denote the time taken by the RW to go from Node $i$ to the only absorbing state, Node 3. Also, let ${ }_{i} \bar{e}=E\left[{ }_{i} \bar{T}\right],{ }_{i} \bar{s}=E\left[{ }_{i} \bar{T}^{2}\right]$, and $V\left({ }_{i} \bar{T}\right)={ }_{i} \bar{v}={ }_{i} \bar{s}-{ }_{i} \bar{e}^{2}$. From Fig. 5, we have $\bar{T}={ }_{0} \bar{T}=1+{ }_{1} \bar{T}$, and the following inter-relations:

$$
{ }_{1} \bar{T}=\left\{\begin{array}{ll}
1+{ }_{1} \bar{T}^{\prime} & \text { with probability } 1 / 3 \\
1+{ }_{2} \bar{T} & \text { with probability } 2 / 3
\end{array} ; \quad{ }_{2} \bar{T}= \begin{cases}1+{ }_{2} \bar{T}^{\prime} & \text { with probability } 2 / 3 \\
1 & \text { with probability } 1 / 3\end{cases}\right.
$$

where ${ }_{1} \bar{T}^{\prime}$ is a RV IID to ${ }_{1} \bar{T}$, and ${ }_{2} \bar{T}^{\prime}$ is a RV IID to ${ }_{2} \bar{T}$. Taking expectations in (1) and solving, we get ${ }_{2} \bar{e}=3,{ }_{1} \bar{e}=9 / 2$. Likewise, first squaring, then taking expectations in (1), and using ${ }_{2} \bar{e}=3,{ }_{1} \bar{e}=9 / 2$, we get ${ }_{2} \bar{s}=15,{ }_{1} \bar{s}=27$. Hence, ${ }_{1} \bar{v}=V\left[{ }_{1} \bar{T}\right]={ }_{1} \bar{s}-{ }_{1} \bar{e}^{2}=27 / 4$. Finally, from $\bar{T}=1+{ }_{1} \bar{T}$, we get $E[\bar{T}]=1+{ }_{1} \bar{e}=11 / 2, V[\bar{T}]=V\left[{ }_{1} \bar{T}\right]=27 / 4=6.75$, and $\mathrm{SD}(\bar{T})=2.5981$.

Indeed, a careful look at Fig. 5 [or equivalently, at the system of equations (1)] also reveals the following more informative result, which resembles the celebrated coupon collection problem. 
Proposition 2.2.2 For a tetrahedron, the cover time $\bar{T}$ is distributed as $1+G_{1}+G_{2}$, where $G_{1}$ and $G_{2}$ are independent geometric $R V$ s with success probabilities $2 / 3$ and $1 / 3$ respectively. In particular, $\bar{T}$ has mean $11 / 2$, variance $27 / 4$ and $S D 2.5981$.

\subsection{Return After Visiting All Vertices on a Tetrahedron}

According to Proposition 2.2.1, the last vertex visited is equally likely to be any one of the three non-origin vertices. Hence, ${ }_{L} T_{R}$, the time to return to origin after visiting all vertices, has the same distribution as ${ }_{1} T_{0}$, which was shown in Subsection 2.1 to be a geometric $(1 / 3) \mathrm{RV}$. Likewise, ${ }_{L} N_{R}$, the number of vertices visited during the return to origin after visiting all vertices, has the same distribution as $N_{R}$, which is uniformly distributed over $\{1,2,3\}$, according to Proposition 2.1.2.

\section{SRW on an Octahedron}

In Fig. 2, since pairs of opposite vertices $(0,5),(1,4),(2,3)$ are non-adjacent, the graph of an octahedron is incomplete. This incompleteness makes the SRW on an octahedron, compared to that on a tetrahedron, relatively more difficult to study. Nonetheless, because every vertex of an octahedron has exactly four adjacent vertices and exactly one vertex at a distance of two, the techniques described in the previous section are still fruitful here, However, the derivations are more complex.

\subsection{Return to Origin on an Octahedron}

To study $T_{R}$, it suffices to keep track of the distance of the current vertex from the origin, and forget the set of vertices already visited. Fig. 6 shows the transition diagram.
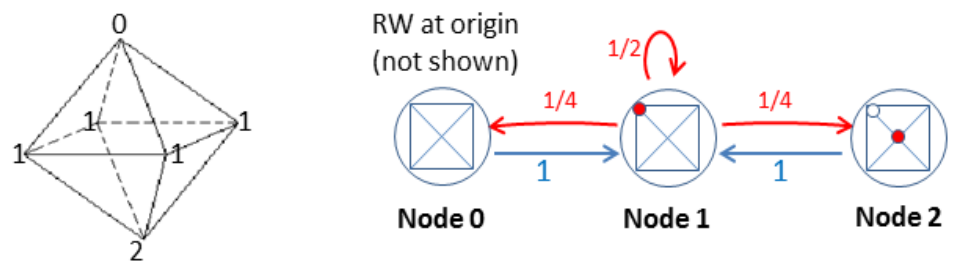

Figure 6. Distance from the origin defines the nodes. One-step transition probabilities between nodes help study $T_{R}$ for an octahedron

We already reasoned that $T_{R}=1+{ }_{1} T_{0}$. By conditioning on the next move, we get

$$
{ }_{1} T_{0}= \begin{cases}1 & \text { with probability } 1 / 4 \\ 1+{ }_{1} T_{0}{ }^{\prime} & \text { with probability } 1 / 2 \\ 2+{ }_{1} T_{0}{ }^{\prime \prime} & \text { with probability } 1 / 4\end{cases}
$$

where ${ }_{1} T_{0},{ }_{1} T_{0}^{\prime},{ }_{1} T_{0}^{\prime \prime}$ are IID RVs. Taking expectations in (2) and solving, we get ${ }_{1} e_{0}=5$. Likewise, squaring, taking expectations in (2), and using ${ }_{1} e_{0}=5$, we get ${ }_{1} s_{0}=47$. Hence, ${ }_{1} v_{0}=47-5^{2}=22$. Also, the distribution of ${ }_{1} T_{0}$ is as follows.

Proposition 3.1.1 For an octahedron, the time $T_{R}$ to return to origin is distributed as $T_{R}=1+{ }_{1} T_{0}$, where ${ }_{1} T_{0}$ has the probability distribution given by

$$
P\left\{{ }_{1} T_{0}=k\right\}=\frac{1}{2 \sqrt{5}}\left[\left(\frac{1+\sqrt{5}}{4}\right)^{k}-\left(\frac{1-\sqrt{5}}{4}\right)^{k}\right], \quad \text { for } k=1,2,3, \ldots
$$


Also, $T_{R}=1+{ }_{1} T_{0}$ has mean 6 , variance 22 and $S D 4.6904$.

Proof. The RW leaves the origin to go to Vertex 1 (renumbered, if needed). By conditioning on the next move out of Vertex 1, we have

$$
\begin{aligned}
P\left\{{ }_{1} T_{0}=1\right\} & =1 / 4 ; \quad P\left\{{ }_{1} T_{0}=2\right\}=1 / 8 ; \quad \text { and thereafter inductively } \\
P\left\{{ }_{1} T_{0}=k+2\right\} & =(1 / 2) P\left\{{ }_{1} T_{0}=k+1\right\}+(1 / 4) P\left\{{ }_{1} T_{0}=k\right\}, \quad \text { for } k=1,2, \ldots
\end{aligned}
$$

We solve the homogeneous recursive relation in (4) by the method of characteristic equation (see, for example, [5]). Here, the characteristic equation $4 x^{2}-2 x-1=0$ has two distinct roots $(1+\sqrt{5}) / 4$ and $(1-\sqrt{5}) / 4$. Using these roots and the boundary conditions in (4), we establish (3).

Q.E.D.

Remark 2. Although the expression on the right hand side of (3) involves radicals, just as one would anticipate from (4), the expression reduces to a rational fraction.

To study $N_{R}$, as shown in Fig. $7($ a), we need a more elaborate transition diagram. Here we keep track of all elements of the triplet - the origin, the current vertex and the pattern of visited vertices. But we treat nodes that are rotations and/or reflections of one another as identical. We draw the one-step transitions until the RW on an octahedron returns to origin. Here, each arrow represents a conditional probability of 1/4. Again, in this diagram the origin is not shown; etc. Also, we invite the reader to interpret this diagram in the same way we did Fig. 4(a).

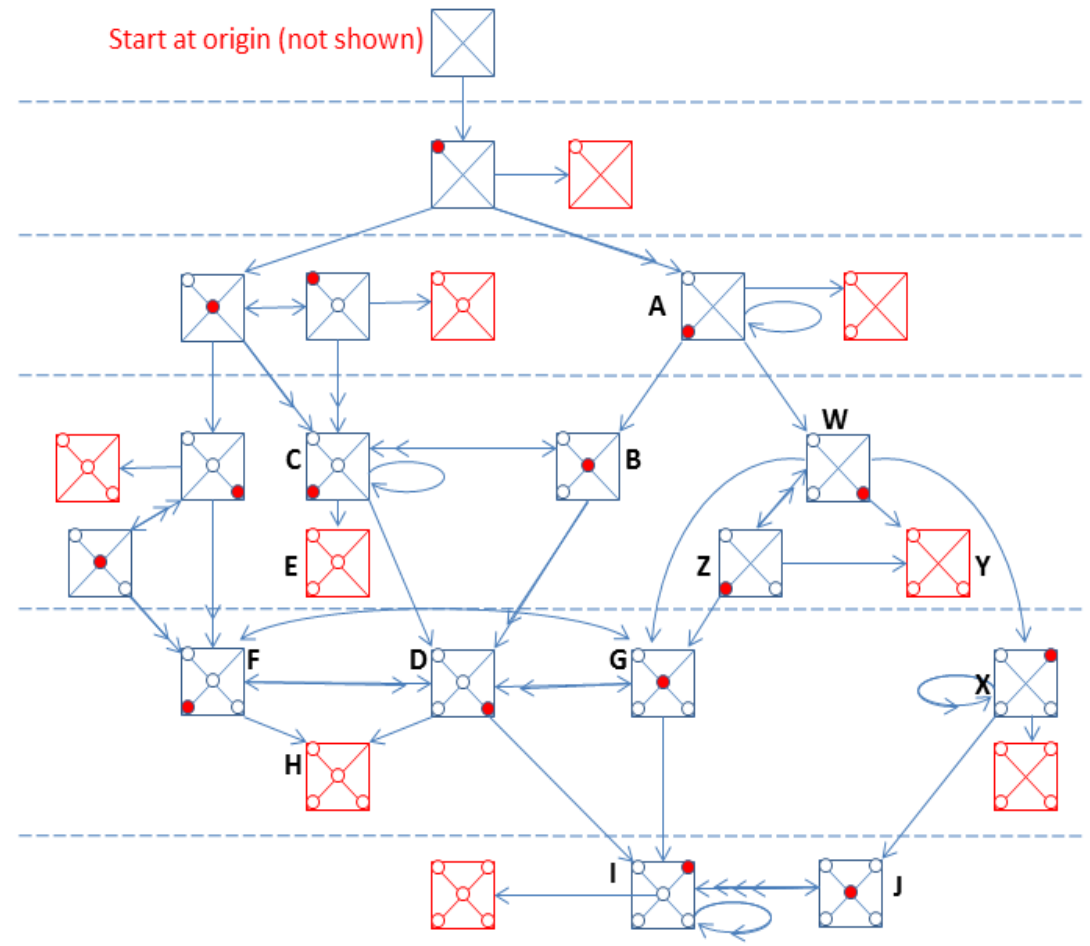

Figure 7(a): One-step transitions until return to origin, help study $N_{R}$ for an octahedron (conditional probability is $1 / 4$ on each arrow)

We invite the readers to either verify the next two diagrams and associated calculations, or contact the corresponding author for details. In Fig. 7(b), we draw the eventual 
transitions after eliminating the loops and interchanges. Thereafter, in Fig. 7(c), by first multiplying the conditional probabilities along all distinct directed pathways from the start to any absorbing node, and then adding up these products, we obtain the probability of reaching that absorbing state. These absorbing nodes are labeled as $\mathrm{R}_{*, *}$, where the first subscript is the value of $N_{R}$ and the second subscript is simply a serial number to distinguish such nodes. Between horizontal separating lines in Fig. 7(c), the values of $N_{R}$ are listed on the left margin, and the associated probabilities on the right margin. Hence, we have proved the following result.

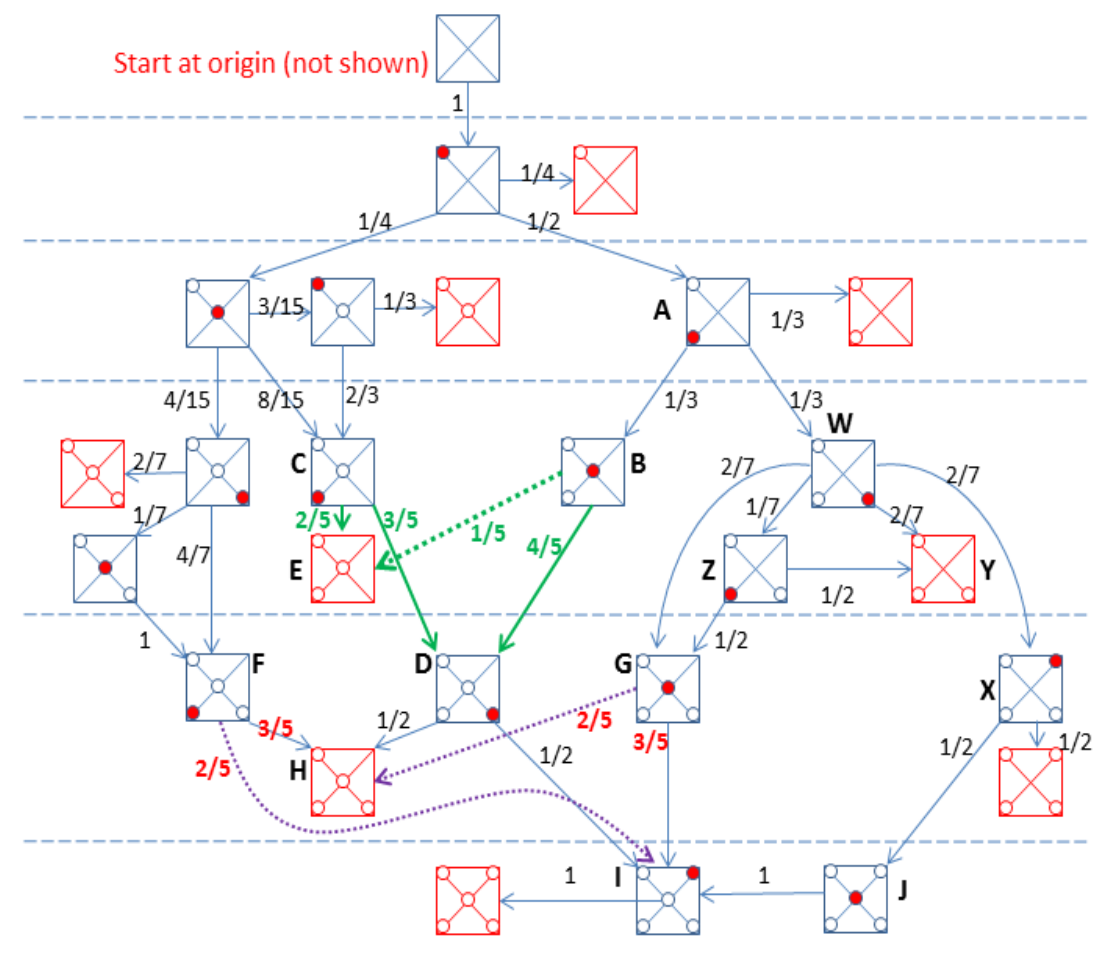

Figure 7(b): Eventual transitions until return to origin, helps study $N_{R}$ for an octahedron (conditional probability shown on each arrow)

Proposition 3.1.2 For an octahedron, the number $N_{R}$ of vertices visited before return to origin has a probability distribution shown in Fig. $7(c)$ and also given in the first row of Table 1. In particular, $N_{R}$ has mean 2.9 , variance $15 / 7=2.1429$ and $S D 1.46385$.

Remark 3. As in Remark 1, without first studying the cover time $\bar{T}$ for an octahedron, we can determine which event will happen first - returning to origin, or visiting all vertices. Indeed, we have $P\left\{T_{R}>\bar{T}\right\}=P\left\{N_{R}=5\right\}=41 / 210=.1952$, and $P\left\{T_{R}<\bar{T}\right\}=$ $P\left\{N_{R}<5\right\}=169 / 210=.8048$.

\subsection{Visiting All Vertices on an Octahedron}

The reader may imagine that there is a cookie at each vertex of the octahedron; and a cookie monster eats each cookie when it visits that vertex for the first time. Then $\bar{T}$ is the number of steps needed until all six cookies are eaten, and $L=X(\bar{T})$ denotes which cookie is eaten the last. 


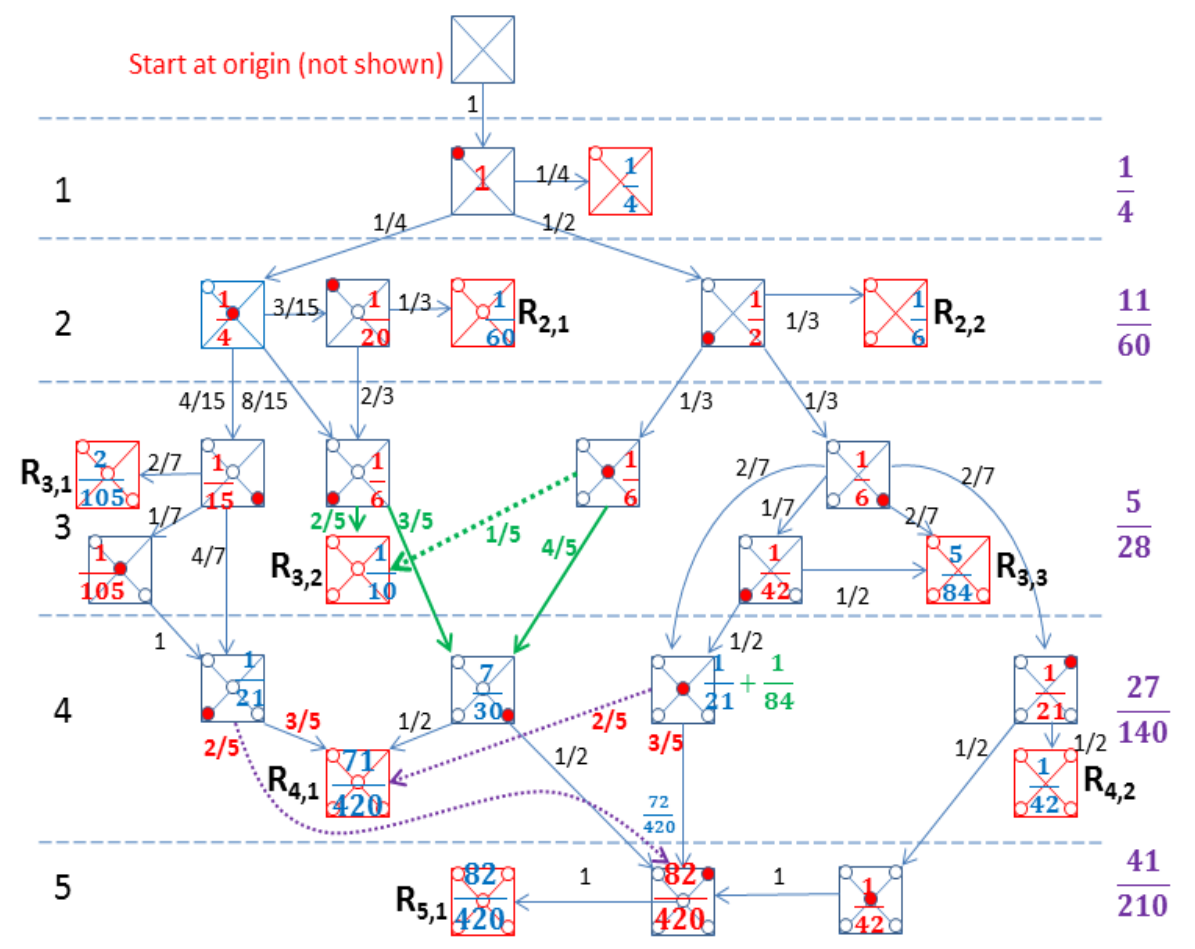

Figure 7(c): The distribution of $N_{R}$ for an octahedron (shown on the right margin) is obtained by adding the probabilities of absorbing nodes (marked $\mathbf{R}_{*, *}$ ) corresponding to each value of $N_{R}$ (shown on the left margin)

Invoking symmetry, we see that $L$ is equally likely to be any one of Vertices $1,2,3,4$ (which are adjacent to the origin) with probability $\alpha$ each; and $L$ is likely to be Vertex 5 (which is opposite the origin) with probability $\beta$. Clearly then, $4 \alpha+\beta=1$, and

$$
\beta=P\{L=5\}=P\left\{L_{1}=5\right\}+P\left\{L_{1}=0, S L_{1}=5\right\}
$$

where $L_{1}$ denotes the last vertex visited and $S L_{1}$ denotes the second last vertex visited, if the RW were to start from Vertex 1 . To justify (5), we argue as follows: If $L_{1}=5$, then by augmenting Vertex 0 at the very beginning of this RW (which starts from Vertex 1 ) would make $L=5$. On the other hand, if $L_{1}=0$, then doing the same thing would make $L=S L_{1}=5$. Since Vertex 5 is adjacent to Vertex 1 , we have $P\left\{L_{1}=5\right\}=\alpha$. A careful investigation shows that $P\left\{L_{1}=0, S L_{1}=5\right\}$ equals the eventual probability that the RW ever reaches Node J of Fig. 7(b). This eventual probability has been already calculated in Fig. $7(\mathrm{c})$ to be $1 / 42$. Hence, from (5), we have $\beta=\alpha+1 / 42$. Finally, $4 \alpha+(\alpha+1 / 42)=1$, implies the following result.

Proposition 3.2.1 For an octahedron, the $R W$ starting at Vertex 0 (origin) visits the last any one of the adjacent Vertices 1, 2, 3, 4 with probability $\alpha=41 / 210$ each; and it visits the last the opposite Vertex 5 with probability $\beta=46 / 210$.

Remark 4. If we focus on a RW that begins at Vertex 1, then clearly $P\left\{L_{1}=0\right\}=\alpha$. But now if we augment $X(-1)=0$ at the very beginning of this RW (which starts at Vertex 1), we reconstruct another RW which starts at Vertex 0 and then without loss of generality moves to Vertex 1 . Then $\left\{L_{1}=0\right\}$ means that the new RW has visited all vertices before returning to the origin. Therefore, $P\left\{L_{1}=0\right\}=P\left\{N_{R}=5\right\}$. The final expression is already calculated in Fig. 7 (c) to be $41 / 210$. 
As in the case of a tetrahedron, to study $\bar{T}$ for the octahedron, we only need to keep track of the pattern of visited vertices and the current vertex, and we can safely forget the original labeling of the vertices (including the origin). In Fig. 8(a), we draw the onestep transitions until all vertices are visited at least once. Here, each arrow represents a probability of $1 / 4$.

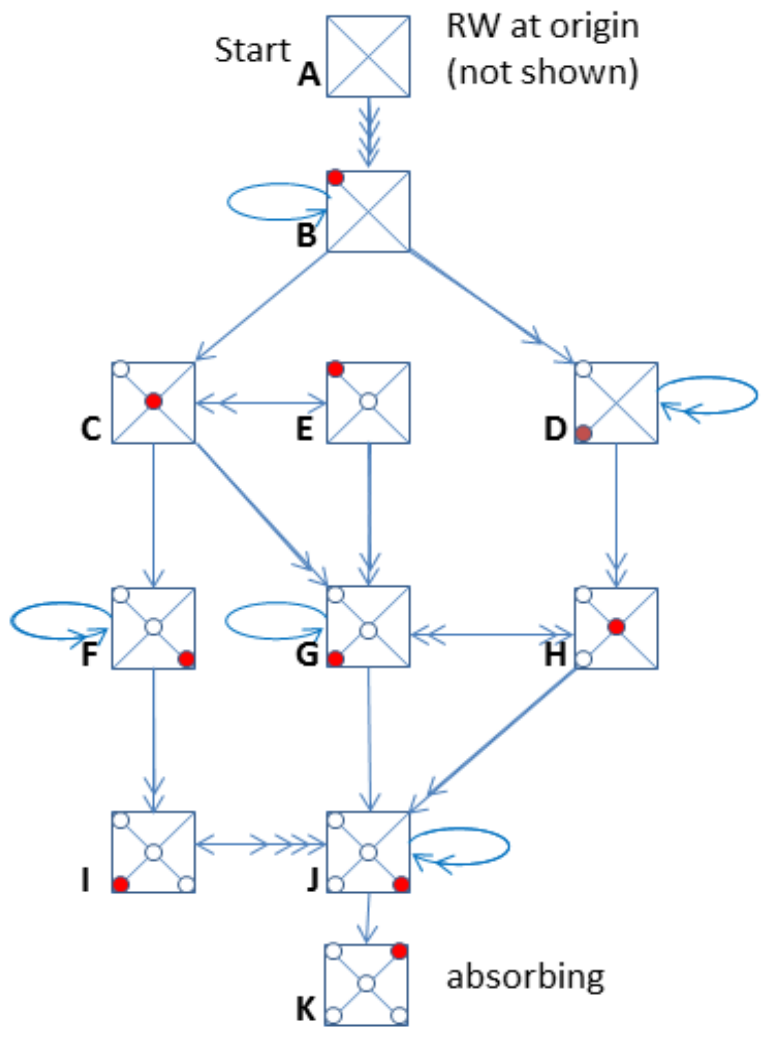

Figure 8(a): One-step transitions until visit all vertices, help study $\bar{T}$ for an octahedron (1/4 prob. on each arrow)

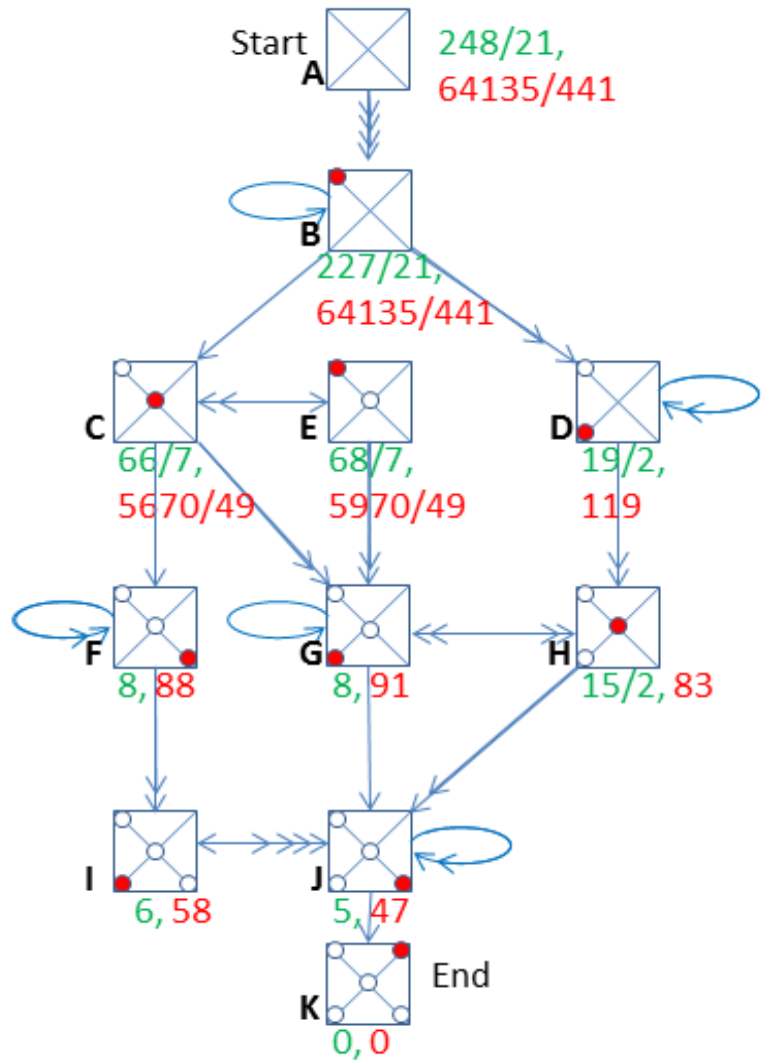

Figure 8(b): Mean time and mean squared time to reach node $\mathrm{K}$ starting from each node, for an octahedron

For any Node Z (ranging over Nodes A through K) in Fig. 8(a), let ${ }_{Z} T_{K}$ denote the time taken by the RW to go from Node $Z$ to Node $K$. We take liberty to re-use a similar notation already used earlier, but we are now referring to transition from one node to another, and not from one vertex to another. We will not attempt to get explicit expressions for the probability distributions of the time variables ${ }_{Z} T_{K}$ 's. However, using Fig. 8(a) we can develop interrelations among the means and the mean squares of ${ }_{Z} T_{K}$ 's. These relations can be solved backwards (receding from Node K), until we evaluate the mean and the mean square of $\bar{T}={ }_{A} T_{K}$. Fig. 8(b) shows the computations. Specifically, the following result holds.

Proposition 3.2.2 For an octahedron, starting from Vertex 0 (origin), the cover time $\bar{T}$ has mean 11.8095 , variance 28.5250 and SD 5.3465 .

\subsection{Return After Visiting All Vertices on an Octahedron}

The distribution of ${ }_{L} T_{R}$, of course, depends on $L$, the last vertex visited. Recall that $L$ is adjacent to the origin (with probability $4 \alpha$ ), or it is opposite the origin (with probability $\beta)$. Accordingly, we have the following result. 
Proposition 3.3.1 For an octahedron, starting from the last visited Vertex L, the time ${ }_{L} T_{R}$ to return to origin has the same probability distribution as ${ }_{1} T_{0}$ with probability $4 \alpha$; and as $1+{ }_{1} T_{0}$ with probability $\beta$, where the distribution of ${ }_{1} T_{0}$ is already described in (3). In particular, ${ }_{L} T_{R}$ has mean 5.2190, variance 22.1711 and $S D 4.7086$.

Likewise, the distribution of ${ }_{L} N_{R}$ also depends on $L$. When $L$ is adjacent to the origin (that is, $L \neq 5$ ), ${ }_{L} N_{R}$ has the same distribution as that of $N_{R}$, the number of distinct vertices among $A=\left\{X(1), X(2), \ldots, X\left(T_{R}-1\right)\right\}$, which is given in Subsection 3.1. But if $L=5$, then ${ }_{L} N_{R}$ has the same distribution as a slightly different RV $M_{R}$, the number of distinct vertices among $\{5\} \cup A$. That is, if one element of $A$ is Vertex 5 , then $M_{R}$ is the number of distinct vertices in $A$; but if none of the elements of $A$ is Vertex 5 , then $M_{R}$ is one more than the number of distinct vertices in $A$. If we denote by $F$ the event that starting from Vertex 1, the RW visits Vertex 5 before it returns to origin, then

$$
P\left\{M_{R}=k\right\}=P\left\{N_{R}=k, F\right\}+P\left\{N_{R}=k-1, F^{c}\right\} \quad \text { for } k=2,3,4,5
$$

The two terms on the right hand side of (6) can be found from Fig. 7(c) by separating the two groups of absorbing nodes - those for which the RW visits Vertex 5 before returning to origin (that is, Nodes $\left\{R_{2,1}, R_{3,1}, R_{3,2}, R_{4,1}, R_{5,1}\right\}$ ), and those for which it does not (that is, Nodes $\left.\left\{R_{2,2}, R_{3,3}, R_{4,2}\right\}\right)$.

The distribution of ${ }_{L} N_{R}$ is obtained by taking the weighted average of the distributions of $N_{R}$ and $M_{R}$, with weights $4 \alpha=82 / 105$ and $\beta=23 / 105$ respectively. Or,

$$
P\left\{{ }_{L} N_{R}=k\right\}=4 \alpha P\left\{N_{R}=k\right\}+\beta P\left\{M_{R}=k\right\} \text { for } k=1, \ldots, 5
$$

We evaluate (6) and (7) in Table 1; and we obtain the following result.

Table 1: For an octahedron, the probability distribution of ${ }_{L} N_{R}$, the number of vertices visited while the RW returns to origin starting after visiting all vertices

\begin{tabular}{r|ccccc|c}
\hline$k$ & 1 & 2 & 3 & 4 & 5 & total \\
\hline$P\left\{N_{R}=k\right\}$ & $\frac{1}{4}$ & $\frac{11}{60}$ & $\frac{5}{28}$ & $\frac{27}{140}$ & $\frac{41}{210}$ & 1 \\
\hline$P\left\{N_{R}=k, F^{c}\right\}$ & $\frac{1}{4}$ & $\frac{1}{6}$ & $\frac{5}{84}$ & $\frac{1}{42}$ & 0 & $\frac{1}{2}$ \\
$P\left\{N_{R}=k, F\right\}$ & 0 & $\frac{1}{60}$ & $\frac{5}{42}$ & $\frac{71}{420}$ & $\frac{41}{210}$ & $\frac{1}{2}$ \\
\hline$P\left\{M_{R}=k\right\}$ & 0 & $\frac{4}{15}$ & $\frac{2}{7}$ & $\frac{8}{35}$ & $\frac{23}{105}$ & 1 \\
\hline$P\left\{N_{R}=k\right\}$ & $\frac{841}{4410}$ & $\frac{889}{4410}$ & $\frac{891}{4410}$ & $\frac{885}{4410}$ & $\frac{884}{4410}$ & 1 \\
, & .1952 & .2016 & .2020 & .2007 & .2005 & 1.000 \\
\hline
\end{tabular}

Proposition 3.3.2 For an octahedron, starting from the last visited Vertex $L$, the number ${ }_{L} N_{R}$ of vertices visited while returning to origin has the probability distribution given in Table 1. In particular, ${ }_{L} N_{R}$ has mean 3.0095, variance 1.9849 and $S D 1.4089$.

\section{SRW on a Hexahedron}

For the RW on a hexahedron, for the sake of brevity, we just outline the results. We invite the reader to use similar reasonings as in Section 3, and fill-in the details. 


\subsection{Return to Origin on a Hexahedron}

To study $T_{R}$ for a hexahedron, one needs a network of four nodes representing possible distances $0,1,2,3$ of the current vertex from the origin. Accordingly,

$$
\begin{aligned}
& T_{R}=1+{ }_{1} T_{0} \\
& { }_{1} T_{0}= \begin{cases}1 & \text { with probability } 1 / 3 \\
1+{ }_{2} T_{0} & \text { with probability } 2 / 3\end{cases} \\
& { }_{2} T_{0}= \begin{cases}1+{ }_{1} T_{0}, & \text { with probability } 2 / 3 \\
2+{ }_{2} T_{0}{ }^{\prime} & \text { with probability } 1 / 3\end{cases}
\end{aligned}
$$

where ${ }_{2} T_{0}$ and ${ }_{2} T_{0}^{\prime}$ are IID RVs. Substituting (9) in (10), we see that ${ }_{2} T_{0}=2 G$, where $G$ is a geometric(2/9) RV. Next, substituting ${ }_{2} T_{0}=2 G$ in (9), and thereafter further substitution that composite expression in (8), we have the following result.

Proposition 4.1.1 For a hexahedron, the time $T_{R}$ to return to origin has a support set $\{2,4,6, \ldots\}$; and the probabilities are given by $P\left\{T_{R}=2\right\}=1 / 3$, and thereafter

$$
P\left\{T_{R}=2 k+2\right\}=\frac{2}{3} \cdot \frac{2}{9} \cdot\left(\frac{7}{9}\right)^{k-1}, \quad \text { for } k=1,2,3, \ldots
$$

In particular, $T_{R}$ has mean 8, variance 60 , and SD 7.7460 .

Remark 5. It is a pleasant surprise that $E\left[T_{R}\right]=8$, which equals the number of vertices of a hexahedron. For the tetrahedron and the octahedron, similar results were seen in Propositions 2.1.1 and 3.1.1 respectively. Indeed, a similar result holds for every Platonic solid (and also for all regular graphs). The intuition behind this phenomenon is as follows: Since each vertex has the same number of edges incident there, in the long-run (that is, as $t \rightarrow \infty$ ) the RW is equally likely to be at any one vertex; and the expected number of steps between successive visits to any particular vertex is the reciprocal of the probability that the RW is at that vertex.

To study $N_{R}$ for a hexahedron, we invite the reader to construct a transition diagram that keeps track of all three features of the configurations - the origin, the current vertex and the pattern of visited vertices - and show the one-step transitions until return to origin. Then the reader must calculate the eventual transitions probabilities; etc. The probability distribution of $N_{R}$ is summarized in the following result.

Proposition 4.1.2 For a hexahedron, the number $N_{R}$ of vertices visited before return to origin has a probability distribution given in Table 2. In particular, $N_{R}$ has mean 3.4476, variance 4.9096 and SD 2.2158.

Table 2: For a hexahedron, the probability distribution of $N_{R}$

\begin{tabular}{c|ccccccc|c}
\hline$k$ & 1 & 2 & 3 & 4 & 5 & 6 & 7 & total \\
\hline$P\left\{N_{R}=k\right\}$ & $\frac{1}{3}$ & $\frac{1}{12}$ & $\frac{11}{84}$ & $\frac{1121}{13860}$ & $\frac{33343}{263340}$ & $\frac{7639}{65835}$ & $\frac{8483}{65835}$ & 1 \\
,. & .3333 & .0833 & .1310 & .0809 & .1266 & .1160 & .1289 & 1.0000 \\
\hline
\end{tabular}

Remark 6. As in Remarks 1 and 3, without first studying the cover time $\bar{T}$ for a hexahedron, we can determine which event will happen first-returning to origin or visiting all vertices. Recall that $T_{R} \neq \bar{T}$. We have $P\left\{T_{R}>\bar{T}\right\}=P\left\{N_{R}=7\right\}=.128852$, and $P\left\{T_{R}<\bar{T}\right\}=P\left\{N_{R}<7\right\}=.871148$. 


\subsection{Visiting All Vertices of a Hexahedron}

Recall the imagery that there is a cookie at each vertex, and a cookie monster is eating each cookie as it visits that vertex for the first time; etc. Also, recall from Section 1 how the vertices of a hexahedron are numbered. Invoking symmetry, note that $P\{L=1\}=$ $P\{L=2\}=P\{L=3\}=\alpha$, say; and $P\{L=4\}=P\{L=5\}=P\{L=6\}=\beta$, say. Let $P\{L=7\}=\gamma$. Imitating our study of $L$ on an octahedron, let us define

$$
\theta_{k}=P\left\{L_{1}=0, S L_{1}=k\right\} \quad \text { for } k=4,5,6,7
$$

where $L_{1}$ denotes the last vertex visited and $S L_{1}$ denotes the second last vertex visited by a RW starting from Vertex 1, and continuing until all vertices are visited. Again, by symmetry, we see that $\theta_{4}=\theta_{5}$. The following lemma tells us how to use $\theta_{5}, \theta_{6}, \theta_{7}$ to calculate $\alpha, \beta, \gamma$.

Lemma 1 One can calculate $\alpha, \beta, \gamma$ based on $\theta_{5}, \theta_{6}, \theta_{7}$ as follows:

$$
\begin{aligned}
1 & =3 \alpha+3 \beta+\gamma \\
\beta & =\frac{2}{3}\left(\alpha+\theta_{5}\right)+\frac{1}{3}\left(\gamma+\theta_{6}\right) \\
\gamma & =\beta+\theta_{7}
\end{aligned}
$$

More explicitly, $\beta=\left(2+6 \theta_{5}+3 \theta_{6}+\theta_{7}\right) / 14$.

Lemma 1 is proved using the same logic as in the proof of (5). For the sake of brevity, we omit its proof. It only remains to evaluate $\theta_{5}, \theta_{6}, \theta_{7}$. To do so, the reader may simply revise the transition diagram used to study of $N_{R}$ : Whereas earlier we had treated a node as absorbing if the RW reaches Vertex 0, now let us also declare a node as absorbing if the RW reaches Vertex $k$. Consequently, all nodes previously accessible from the newly declared absorbing nodes are completely eliminated, and the diagram is much simplified. By calculating the probabilities of reaching the terminal node(s) for which $\left\{L_{1}=0, S L_{1}=k\right\}$, the reader can verify that

$$
\theta_{5}=\frac{7}{627}, \theta_{6}=\frac{16}{1881}, \theta_{7}=\frac{2}{165}
$$

Then the reader can establish the following result.

Proposition 4.2.1 For a hexahedron, starting from Vertex 0 (origin), the Vertex L visited the last takes values 1,2,3 with associated probability $\alpha=8483 / 65835=0.12885243$; it takes values 4, 5, 6 with probability $\beta=3299 / 21945=0.15033037$; and it takes value 7 with probability $\gamma=713 / 4389=0.16245158$.

Remark \%. As in Remark 4, $\alpha$ can be found using the results of Subsection 4.1 as

$$
\alpha=P\{L=1\}=P\left\{L_{1}=0\right\}=P\left\{T_{R}>\bar{T}\right\}=P\left\{N_{R}=7\right\}=.128852
$$

Once $\alpha$ is known, in view of (13) and (15), it suffices to evaluate only $\theta_{7}$ (and skip evaluating $\theta_{5}$ and $\theta_{6}$ ).

To study $\bar{T}$ for a hexahedron, we need a transition diagram which involves 26 nodes that keep track of the pattern of visited vertices and the current vertex, and safely forgets the original labeling of the vertices (including the origin). The diagram yields interrelations among the means and the mean squares of random times until the last node is reached starting from any of these nodes. Working backwards, the interested reader can obtain the following result. The mean can be found in [1], but the SD is entirely new. 
Proposition 4.2.2 For a hexahedron, the cover time $\bar{T}$ has mean 1996/95 = 21.01, mean square $11468329 / 21660$, variance 88.02819 and SD 9.382334 .

\subsection{Return After Visiting All Vertices on a Hexahedron}

Using (8)-(10) and Proposition 4.2.1, we have the following result.

Proposition 4.3.1 For an octahedron, starting from the last visited Vertex L, the time ${ }_{L} T_{R}$ to return to origin is distributed as ${ }_{1} T_{0}$ with probability $3 \alpha$, as ${ }_{2} T_{0}$ with probability $3 \beta$, and as $1+{ }_{2} T_{0}$ with probability $\gamma$. Here, the distribution of ${ }_{1} T_{0}$ is 1 with probability $1 / 3$, and $1+{ }_{2} T_{0}$ with probability 2/3; and the distribution of ${ }_{2} T_{0}$ is twice a geometric variable with success probability $2 / 9$. In particular, ${ }_{L} T_{R}$ has mean $7+6 \beta+3 \gamma=8.3893$, mean square $109+105 \beta+54 \gamma=133.5571$, variance 63.1761 and $S D 7.9483$.

The distribution of ${ }_{L} N_{R}$, the number of vertices visited during the return to origin after visiting all vertices, of course, depends on $L$. All necessary ingredients are already given above so that the interested reader can construct the exact distribution of ${ }_{L} N_{R}$ by suitably combining these components.

\section{Conclusion}

We have studied SRWs on the vertices of a tetrahedron, an octahedron, and a hexahedron. Specifically, we have studied the distributions (or at least the means and the SDs) of (1) the time until return to origin, (2) the time until all vertices are visited, and (3) the additional time until return to origin after visiting all vertices. Alongside, we also studied the distributions of the last vertex visited and the number of vertices visited during the return to origin after leaving the origin, or after visiting all vertices.

While so far we have talked about SRWs on the vertices of regular polyhedra, we can easily extend the results to SRWs on their faces, simply by using the concept of dual graphs. A dual of a graph is another graph where each face of the old graph becomes a vertex of the new graph (and vice versa), with the understanding that two vertices of the new graph are adjacent if and only if the corresponding faces of the old graph share a common boundary. A tetrahedron is its own dual. An octahedron and a hexahedron are duals of each other. Thus, by studying the SRW on the vertices of a tetrahedron, an octahedron and a hexahedron, we have studied the SRW on the faces of a tetrahedron, a hexahedron and an octahedron respectively.

We invite the reader to complete the study of the SRW on the vertices/faces of the two remaining Platonic solids - icosahedron and dodecahedron - which are duals of each other. Techniques developed here can be beneficial also for studying RWs on higher dimensional regular polytopes and other distance-regular graphs.

Another direction of research is to allow asymmetry in the walk. One form of asymmetry considers the edge along which the RW has reached the current vertex as a special edge labeled 0 ; and the other edges incident at the current vertex are labeled $1,2, \ldots$ going clockwise (from outside). One can then assume that starting from the origin the RW is equally likely to go to any adjacent vertex; but thereafter at each successive step, the RW chooses the next vertex according to a specified one-step transition probability vector $\mathbf{p}=\left(p_{0}, p_{1}, p_{2}, \ldots\right)$, where $p_{h}$ is the probability of traveling along edge $h$. For example, on a tetrahedron an asymmetric RW with $\mathbf{p}=(.2, .4, .4)$ yields the mean and variance 
of $T_{R}$ to be 4 and 16 respectively. The distribution of $N_{R}$ has support set $\{1,2,3\}$ with probability mass function $(.2, .4, .4)$. The last vertex is still equally likely to be any one of

the non-origin vertices. The cover time $\bar{T}$ is one more than the sum of two independent geometric RVs with success probabilities .8 and .4 respectively. Hence, the cover time has mean $1+1.25+2.5=4.75$, variance $5 / 16+15 / 4=65 / 16=4.0625$, and SD 2.0156. Other forms of asymmetric RWs are also possible.

\section{Acknowledgments}

The second author thanks the IUPUI authorities for sponsoring her visit there while this research was done. Both authors thank Prof. Humberto Barreto of DePauw University for catching an error in an earlier manuscript regarding the distribution of $L$ for an octahedron. Special thanks are due to two anonymous referees for their insightful comments, and to the Joint Editors for their guidance and encouragement.

\section{References}

[1] Brady, Z. (aka Zeb) (2011), What is the cover time of a random walk on a cube? http://mathoverflow.net/questions/59244/

[2] Cromwell, P.R. (1997), Polyhedra, New York: Cambridge University Press. MR1458063

[3] Euclid (1956). Heath, T.L., ed. The Thirteen Books of Euclid's Elements, Books X-XIII (2nd unabr. ed.), New York: Dover Publications. MR0075873

[4] Göbel, F. and Jagers, A.A. (1974), Random walks on graphs, Stochastic Processes and their Applications, 102, 311-336. MR0397887

[5] Graham, R.L., Knuth, D.E. and Patashnik, O. (1994). Concrete Mathematics: A Foundation for Computer Science (2 ed.), Boston: Addison-Wesley. MR1679822

[6] Letac, G. and Takács, L. (1980), Random walks on a dodecahedron, J. Appl. Prob., 17, 373-384. MR0568948

[7] Maiti, S.I. and Sarkar, J. (2016), Symmetric random walk on paths and cycles, Submitted.

[8] Sarkar, J. (2006), Random walk on a polygon, In: Recent Developments in Nonparametric Inference and Probability, J Sun, A DasGupta, V Melfi, C Page, Eds., IMS Lecture NotesMonograph Series, 50, 31-43, Beachwood, OH: Inst. Math. Statist. MR 2409062.

[9] van Slijpe, A.R.D. (1984), Random walks on regular polyhedra and other distance-regular graphs, Statistica Neerlandica, 38, 273-292. MR0773043

[10] van Slijpe, A.R.D. (1985), Random walks on the triangular prism and other vertex-transitive graphs, J. Comput. and Applied Math., 15, 383-394. MR0851897

[11] Wildberger, N.J. (2014), Hypergroups associated to random walks on the Platonic solids, web.maths. unsw.edu.au/ norman/papers/RandomWalksPlatonicSolids.pdf 